\title{
GPU ACCELERATED INVERSE PHOTON MAPPING FOR REAL-TIME SURFACE REFLECTANCE MODELING
}

\author{
Takashi Machida†, Naokazu Yokoya \\ $†$ Cybermedia Center, Osaka University, Japan \\ $\ddagger$ Graduate School of Information Science, Nara Institute of Science and Technology, Japan
}

\begin{abstract}
This paper investigates the problem of object surface reflectance modeling, which is sometimes referred to as inverse reflectometry, for photorealistic rendering and effective multimedia applications. A number of methods have been developed for estimating object surface reflectance properties in order to render real objects under arbitrary illumination conditions. However, it is still difficult to densely estimate surface reflectance properties in real-time. This paper describes a new method for real-time estimation of the non-uniform surface reflectance properties in the inverse rendering framework. Experiments are conducted in order to demonstrate the usefulness and the advantage of the proposed methods through comparative study.
\end{abstract}

\section{INTRODUCTION}

Inverse rendering is an effective technique to produce a photorealistic image, object geometry, reflectance properties and lighting effect in a scene. In the fields of computer vision and graphics, a number of methods have been developed to estimate reflectance properties from images $[1,2,3,4]$. If the object surface reflectance properties are estimated at once, the virtualized object can be rendered appropriately under arbitrary illumination conditions.

For estimating surface reflectance properties, it is necessary to estimate the incident radiances of surfaces in the scene because the light that any particular surface receives may arrive not only from the light sources but also from the rest of the environment through indirect illumination. The estimation of incident radiances allow to estimate the reflectance properties of the surfaces in the scene via an iterative optimization procedure, which allows to re-estimate the incident radiances. Loscos et al. [2] and Drettakis et al. [1] have attempted to estimate object surface reflectance properties based on radiosity equations. Yu et al. [5] have estimated surface reflectance properties of a room from color and geometry data considering both diffuse and specular interreflections based on the inverse global illumination rendering. Boivin et al. [3] have also attempted to estimate surface reflectance properties with considering diffuse interreflections.
These methods assume that the surface of interest has uniform reflectance properties. Therefore their algorithms cannot be applied to an non-uniform surface reflectance object.

To resolve these problems, we proposed the inverse photon mapping [6]. The photon mapping, which is famous rendering method in $\mathrm{CG}$, can represent diffuse and specular interreflections based on computing emission of photons from a light. Therefore, the inverse photon mapping can estimate non-uniform reflectance parameters without the influence of both diffuse and specular interreflections. However, there still remains a problem that the inverse photon mapping has much computational time to estimate reflectance properties. In this paper, we propose a new method to rapidly estimate surface reflectance properties by the GPU(Graphics Processing Unit) accelerated photon mapping. There is also a problem that the rendering result by photon mapping has low frequency biased noise. To eliminate the noise, the proposed method has a large number of sampling of rays on GPU.

\section{REFLECTANCE MODELING FROM RANGE AND COLOR IMAGES}

Figure 1 shows a flow diagram of estimating surface reflectance properties. Our process consists of five parts: measurement of an object (A, C), selection of light source (B), initial estimation of reflectance parameters using our previous method [4] (D), and reflectance estimation using GPU accelerated photon mapping (E).

\subsection{Measurement and selection of positions of light source}

We use a laser rangefinder (Cyberware 3030RGB) with known positions of point light sources and a camera for acquiring surface color images, as shown in Figure 2(a). This system can obtain registered range and surface color texture images at the same time by rotating the rangefinder and the camera around an object.

In the present experimental setup, multiple positions of a light are determined among 60 possible positions prepared around the laser rangefinder and these are two-dimensionally arranged at the interval of $5 \mathrm{~cm}$ as shown in Figure 2(b). The 


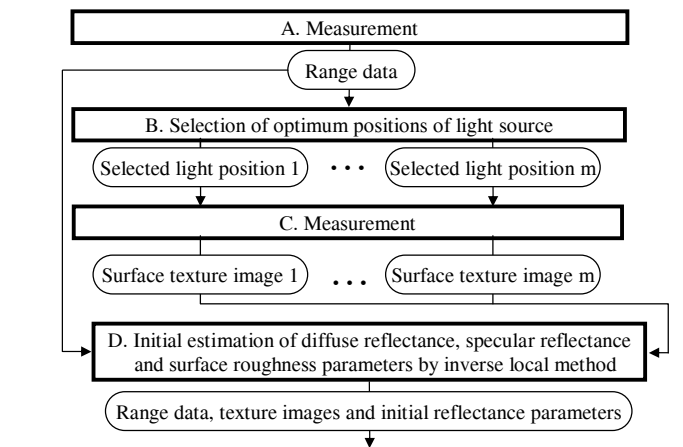

E. Estimation of reflectance parameters based on GPU accelerated photon mapping

Fig. 1. Flow diagram of estimating surface reflectance properties

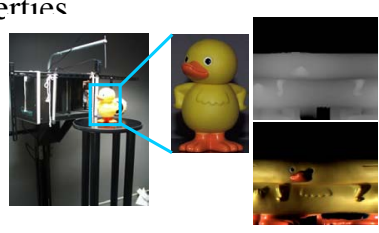

(a) Appearance of

3D-Digitizer and aquired data

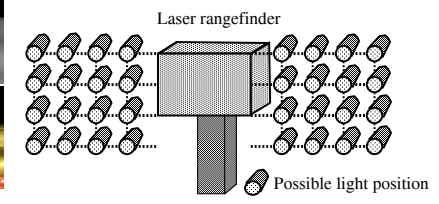

(b) Multiple possible light source positions
Fig. 2. Experimental setup.

positions of a camera and a light source are calibrated in advance. After optimum light positions are selected, a single light is attached at the selected positions in turn so that the calibration of brightness among multiple lights is not needed. We can also ignore the influence of environmental light by measuring the object in a dark room.

Here, we employ the Torrance-Sparrow model [7] to select optimum light positions so that both diffuse and specular reflection components can be densely observed (See [4] for detail). The Torrance-Sparrow model is given as:

$$
i_{x}=\frac{Y}{D^{2}}\left\{P_{d x} \cos \theta_{d x}+\frac{P_{s x}}{\cos \theta_{v x}} \exp \left(-\frac{\theta_{r x}^{2}}{2 \sigma_{x}^{2}}\right)\right\},
$$

where $i_{x}$ represents an observed intensity corresponding to the surface point $x, i_{d x}$ and $i_{s x}$ denote the diffuse and specular reflection components, respectively, $D$ is an attenuation coefficient related to the distance between a point light source and an object surface point, and $Y$ represents the strength of a light source. $P_{d x}, P_{s x}$ and $\sigma_{x}$ are the diffuse reflectance parameter, specular reflectance parameter, and surface roughness parameter, respectively. $\theta_{d x}$ is the angle between light source vector and surface normal vector, $\theta_{v x}$ is the angle between viewing vector and surface normal vector, and $\theta_{r . x}$ is the angle between surface normal vector and half vector. Note that half vector is the vector located halfway between light vector and viewing vector. All vectors are unit vectors.

A texture image is obtained with a selected light position $p(p=1, \cdots, m)$ and consists of $\gamma$ pixels $\left(i_{p 1}, \cdots, i_{p \gamma}\right)$, where $i_{p x}$ means a color intensity of a surface point $x$. Each pixel is classified into three types $T_{\text {diff }}, T_{\text {spec }}$ and $T_{\text {none }}$.
$T_{\text {diff }}$ means a pixel containing only the diffuse reflection component and $T_{\text {spec }}$ means a pixel containing strong specular reflection component. $T_{\text {none }}$ means a pixel which is classified into neither $T_{\text {diff }}$ nor $T_{\text {spec }}$.

\subsection{Accelerated inverse photon mapping for estimation of reflectance parameters}

In this paper, we employ the photon mapping. By using the inverse photon mapping, both diffuse and specular interreflections on the object surface can be taken into account. The photon mapping also does not require tessellated patches due to its pixel base calculations, and thus even if the object has a complicated shape, the photon mapping can render efficiently compared with the radiosity rendering method.

\subsubsection{Photon mapping accelerated by GPU}

In the photon mapping [8], an outgoing radiance $L$ from a surface point $x$ is calculated in order to decide the surface color. The following equations form the rendering equations in the photon mapping.

$$
\begin{aligned}
L(x, \vec{\omega}) & =L^{e}(x, \vec{\omega})+L^{r}(x, \vec{\omega}), \\
L^{r}(x, \vec{\omega}) & =\int_{\Omega} f\left(x, \vec{\omega}^{\prime}, \vec{\omega}\right) L^{o}\left(x, \vec{\omega}^{\prime}\right)\left(\vec{\omega}^{\prime} \cdot \vec{n}\right) d \vec{\omega}^{\prime}, \\
x & : \quad \text { Surface point } \\
\vec{n} & : \quad \text { Unit vector of surface normal at } x \\
\vec{\omega} & : \quad \text { Direction from outgoing radiance } \\
\vec{\omega}^{\prime} & : \text { Direction of incoming radiance } \\
d \vec{\omega} & : \quad \text { Differential solid angle } \\
\Omega & : \text { Hemisphere of directions }
\end{aligned}
$$

where $L^{e}, L^{r}, L^{o}$ and $f$ are the emitted radiance, the reflected radiance, the incoming radiance, and a BRDF (i.e. the Torrance-Sparrow model), respectively.

The photon mapping is two path rendering method. The first path distributes the photon from the light source. The second path gathers the photon on object surfaces. Because the second path includes ray tracing process, it causes much computational time. Here, the proposed method uses a randomly sampled global ray direction. Using this direction, expensive ray intersection can be regarded as a multi-layered parallel projection (see Figure 3). Since graphics hardware can process matrix transformation including parallel projection very fast, such a method is natural and efficient compared with using GPU as a parallel ray tracing processor. Intersection points of global ray direction from sampling points cannot be obtained by single parallel projection, so inverse of depth peeling is used instead to get actual intersection points. Each depth layer is projected from far to near and intersection points sample nearest depth layer. Depth layer texel behind the intersection point is culled by a KILL operation. To reduce the number of depth peeling iterations, criteria based on the number of sampled points can be used to stop the iteration 


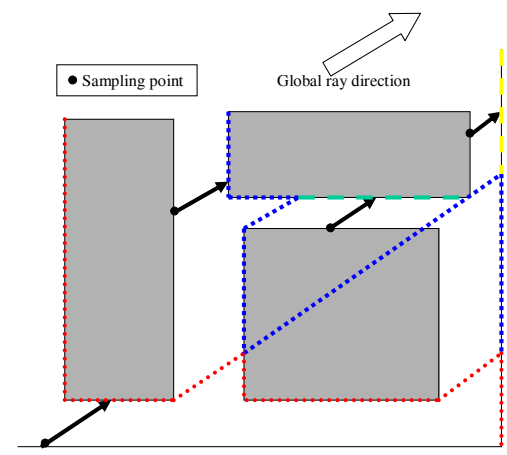

Fig. 3. Relation between depth layer and final gathering.

without significant error on resulting image. Since the proposed method is per-pixel, it can process any form of problem that requires a large number of samples on hemisphere such as precomputation of radiance transfer.

\subsubsection{Iterative estimation of reflectance parameters}

Figure 4 shows the flow diagram of the present method. As an initial estimation, the reflectance parameters are obtained by our previous method base on inverse local rendering [4] which does not consider both diffuse and specular interreflections. Here, let $P_{d x}^{i n i t}, P_{s x}^{i n i t}$ and $\sigma_{x}^{i n i t}$ be the reflectance parameters obtained in this process. These parameters are used as initial parameters. In the next process (E) in Figure 1, our approach uses the GPU accelerated photon mapping as described the previous section. In the following, the detail of reflectance parameter estimation is described.

The reflectance parameter estimation method based on inverse photon mapping is separated into two processes, (a) and (b), as shown in Figure 4. The first process is for the diffuse reflectance parameter estimation ((a) in Figure 4), and the second process is for the estimation of the specular reflectance and surface roughness parameters ((b) in Figure 4). These processes are performed iteratively. In each process, the following equation is minimized at each pixel in the texture image:

$$
\begin{aligned}
\widehat{i_{p x}} & =I_{p x}\left\{\frac{P_{d x}}{\pi}+P_{s x} K\left(\theta_{v x}, \theta_{r x}, \sigma_{x}\right)\right\} \\
E\left(P_{d x}, P_{s x}, \sigma_{k}\right) & =\sum_{p=1}^{q}\left(i_{p x}-\widehat{i_{p x}}\right)^{2}
\end{aligned}
$$

where $i_{p x}$ is the measured radiance (color intensity) at surface point $x$ with light source position $p, \widehat{i_{p x}}$ is the irradiance that is computed from Equation (1) at surface point $x$ with light source position $p$. $K\left(\theta_{v x}, \theta_{r x}, \sigma_{x}\right)$ denotes the specular term in Equation (1) and is a non-linear equation, and the other parameters are the same as in Equation (1). $q$ denotes the number of selected light positions at surface point $x$ in categories $T_{\text {diff }}, T_{\text {spec }}$ and $T_{\text {none }}$ among the selected light positions $m$.

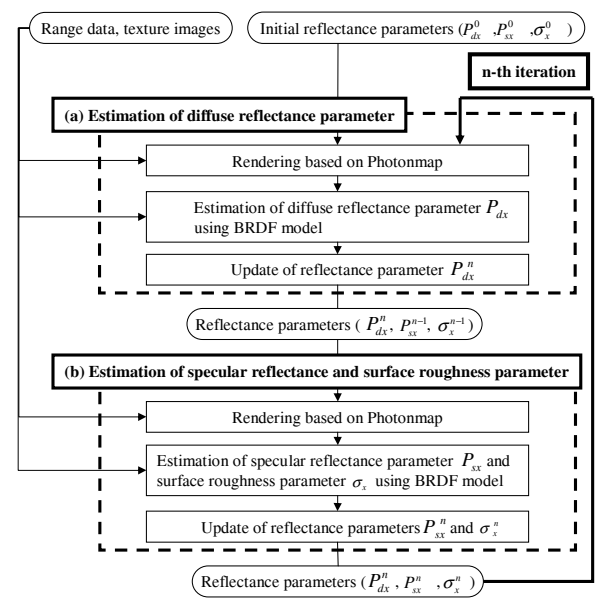

Fig. 4. Flow diagram of estimating surface reflectance properties based on inverse photon mapping.

\section{EXPERIMENTAL RESULTS}

In experiments, we compare the proposed method with our previous method [4, 6] (hereinafter referred to as Method I and II). It should be noted that Method I is the inverse local mapping which does not consider interreflections. Method II is the inverse photon mapping without GPU. Method III is the present method which uses the GPU accelerated photon mapping. A standard PC (Pentium 4, $3.06 \mathrm{GHz}$, memory: 2 GB, GPU: ATI Radeon X800) is used. The number of photons is 2 million. We use Objects A and B shown in Figures 5(a), (b). These objects have non-uniform or uniform diffuse and specular reflectance properties.

Figure 5(c) shows the number of selected light, average and the variance of differences between real and synthetic images, and the computational time. The numbers in bracket in the selected light mean the number of light sources to densely observe the diffuse reflection component. The measurability of both diffuse and specular reflection components is observed about $90 \%$ of the whole object, and linear interpolation is conducted when the specular reflectance and the surface roughness parameters can not be estimated. Method II and III has much smaller variances than Method I for all the objects. These results show Method II and III can accurately estimate each reflectance parameter even if diffuse and specular interreflections occur. In computational time of Method II and III, the numbers in bracket mean the calculation time to estimate reflectance parameters by the inverse photon mapping. Method III is much lower computational time than Method II and the error of Method III is as much as Method II.

Figure 6 shows the cylindrical images of real objects and difference images (modified brightness) between real and synthetic images (rendered by photon mapping) in Method II and III for each object, respectively. Synthetic images are rendered using estimated reflectance parameters under the same illumination condition as in the real images. The errors are 


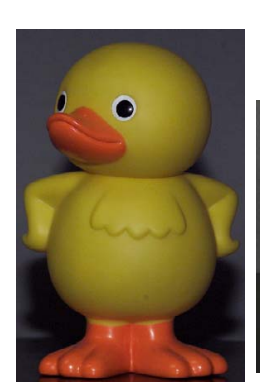

(a) Object $\mathrm{A}$

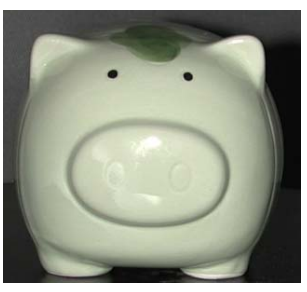

(b) Object B

\begin{tabular}{|c|c|c|l|l|l|}
\hline & $\begin{array}{c}\text { The number of } \\
\text { Object }\end{array}$ & Method & Average & Variance & Time [h:m] \\
\hline \hline \multirow{3}{*}{ A } & \multirow{3}{*}{$12(5)$} & Method I & 30.3 & 709.1 & $0: 05$ \\
& & Method II & 0.99 & 5.5 & $1: 27(1: 22)$ \\
& & Method III & 1.0 & 5.6 & $0: 17(0: 12)$ \\
\hline \multirow{3}{*}{ B } & \multirow{3}{*}{$10(3)$} & Method I & 21.7 & 653.3 & $0: 06$ \\
& & Method II & 0.44 & 3.3 & $1: 38(1: 32)$ \\
& & Method III & 0.49 & 3.3 & $0: 12(0: 06)$ \\
\hline
\end{tabular}

(c) Comparison of the differences between real and synthetic images and computational costs of three methods.

Fig. 5. Three objects used in experiment and measurability of both reflection components.

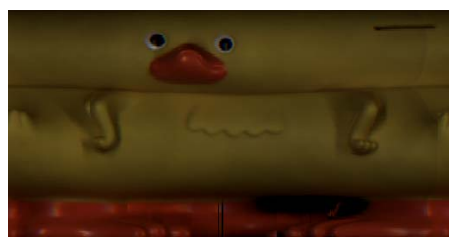

(a) Real image

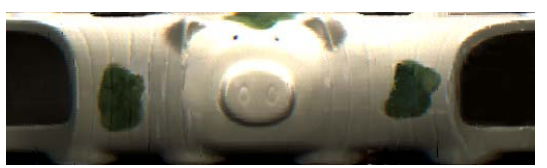

(a) Real image

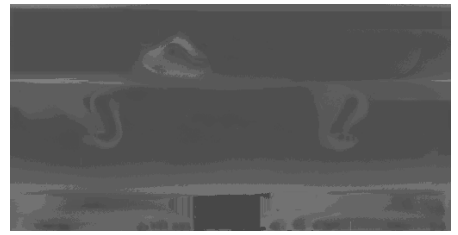

(b) Difference between real and synthetic images by Method II

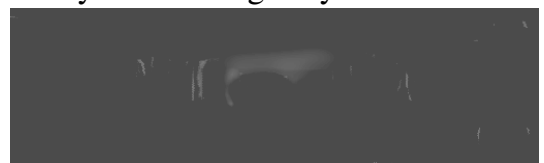

(b) Difference between real and synthetic images by Method II

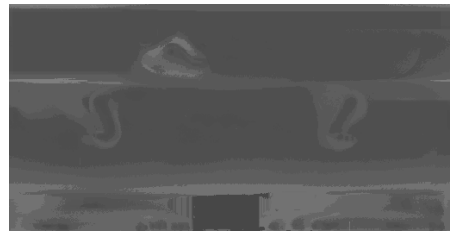

(c) Difference between real and synthetic images by Method III

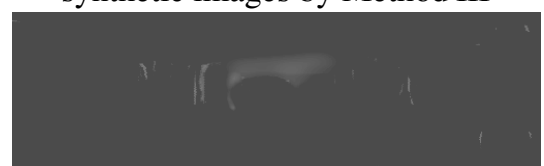

(c) Difference between real and synthetic images by Method III

Fig. 6. A comparison of differences between real and synthetic images for Object A and B.

caused by quantization errors in range data and calculation of photon mapping. The numbers of iteration in estimation of Object A and B are 33 and 35, respectively. It ordinarily takes $30 \sim 40$ iterations to estimate reflectance parameters.

\section{CONCLUSIONS}

In this paper, we have proposed a new method for densely estimating non-uniform reflectance properties of real objects. In our approach, we employ the photon mapping accelerated by GPU which can represent all of the lighting effects in the real world. This method is independent of complexity of the object geometry unlike the radiosity rendering method. Therefore, the inverse photon mapping method can be applied to objects with various reflectance properties. In future work, we will synthesize a virtualized object into a real world to construct a mixed environment. We will also be concerned with more rapid estimation by implementing the parallel algorithm on GPU, as real-time estimation has significant benefit in applying computer vision and graphics.

\section{REFERENCES}

[1] G. Drettakis, L. Robert, and S. Bougnoux, "Interactive Common Illumination for Computer Augmented reality," Proc. Eouro- graphics Rendering Workshop 1997, pp. 45-56, June 1997.

[2] C. Loscos, G. Drettakis, and L. Robert, "Interactive Virtual Relighting of Real Scenes," IEEE Trans. Visualization and Computer Graphics, vol. 6, no. 4, pp. 289-305, December 2000.

[3] S. Boivin and A. Gagalowicz, "Image-Based Rendering of Diffuse, Specular and Glossy Surfaces from a Single Image," Proc. ACM SIGGRAPH '01, pp. 107-116, August 2001.

[4] T. Machida, N. Yokoya, and H. Takemura, "Surface Reflectance Modeling of Real Objects with Interreflections for Augmented Virtuality," Color Research and Application, vol. 31, no. 1, pp. 38-47, February 2006.

[5] Y. Yu, P. E. Debevec, J. Malik, and T. Hawkins, "Inverse Global Illumination: Recovering Reflectance Models of Real Scenes from Photographs," Proc. ACM SIGGRAPH '99, pp. 215-227, August 1999.

[6] T. Machida, N. Yokoya, and H. Takemura, "Inverse global illumination rendering for dense estimation of surface reference properties," Proc. 14th Scandinavian Conf. on Image Analysis (SCIA2005), pp. 790-801, June 2005.

[7] K. E. Torrance and E. M. Sparrow, "Theory for Off-specular Reflection from Roughened Surfaces," Journal of the Optical Society of America, vol. 57, no. 9, pp. 1105-1114, 1967.

[8] H. W. Jensen, Realistic Image Synthesis Using Photon Mapping, A K Peters, Ltd, 1st edition, 2001. 